\title{
CORRESPONDENCE
}

\section{Estimation bias resulting from sparse data: response to comments by Zhang and Ren}

\author{
F. Pappalardo * (1) and G. Landoni
}

@ 2016 Springer-Verlag Berlin Heidelberg and ESICM

We thank Drs. Zhang and Ren [1] for their interest in our study [2]. After initial promising findings [3] we performed the first randomized controlled trial (RCT) on protein $\mathrm{C}$ zymogen in adult septic patients. We found no difference in the primary composite endpoint (prolonged ICU stay and/or 30-day mortality) and an alarming signal towards an increased ICU mortality (79 vs $39 \%$, $p=0.02)$ and hospital mortality ( 84 vs $44 \%, p=0.01$ ) in the study group that was confirmed by Kaplan-Meier $(p=0.035)$ and a multivariate analysis, where protein $\mathrm{C}$ zymogen was the only independent predictor of ICU mortality $(p=0.011)$.

We agree with Zhang and Ren that the study was underpowered to reach definitive conclusions about survival in the study population, but the absence of a beneficial effect on the primary outcome was clear and the baseline clinical characteristics were not different between groups. We confirm that the findings of our study clearly suggest not to use this expensive drug in septic patients. We acknowledge that this result might be flawed by some statistical limitation due to 'estimation bias from sparse data'; however, the clinical impact of our findings is so striking and meaningful that study termination was definitely necessary. Further RCTs must be performed before considering the use of protein $\mathrm{C}$ zymogen in pediatric or adult septic patients.

\section{Acknowledgments \\ The study received a grant from the Italian Ministry of Health (Grant 2009-1607350).}

Accepted: 29 August 2016

Published online: 27 September 2016

References

1. Zhang Z, Ren B (2016) Estimation bias resulting from sparse data. Intensive Care Med. doi:10.1007/s00134-016-4495-0

2. Pappalardo F, Crivellari M, Di Prima AL et al (2016) Protein C zymogen in severe sepsis: a double-blinded, placebo controlled, randomized study. Intensive Care Med. doi:10.1007/s00134-016-4405-5

3. Crivellari M, Della Valle P, Landoni $G$ et al (2009) Human protein C zymogen concentrate in patients with severe sepsis and multiple organ failure after cardiac surgery. Intensive Care Med 35:1959-63
*Correspondence: pappalardo.federico@hsr.it

San Raffaele Scientific Institute, Vita-Salute University, Milan, Italy

This reply refers to the comment available at doi:10.1007/s00134-016-4495-0. 